Francisco Julián Durazo Herrmann*

\title{
MÉXICO Y LA APACHERÍA
}

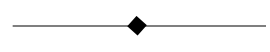

\section{RESU M E N}

El propósito de este artículo es analizar, desde la perspectiva de la polítca exterior, las relaciones del gobierno mexicano con los apaches, una de las tribus nómadas que poblaban el septentrión mexicano. La tesis que sustenta es que, a pesar de que la política de México hacia los apaches no fue internacional ni en su objeto (los apaches nunca fueron tratados como una nación independiente) ni en sus instrumentos (la política apache fue diseñada y ejecutada principalmente por autoridades locales y estatales), esta política sí tenía un objetivo claro, aunque indirecto, de política exterior: consolidar el control mexicano sobre su frontera con los Estados Unidos.

\section{A BSTRACT}

The purpose of this document is to analyze the relations between the government of Mexico and the A paches, one of the nomadic tribes inhabiting N orthern M exico, with the tools and from the perspective of foreign policy. My hypothesis is that, although Mexico's policy towards the A paches was never international neither in its object (as the A paches were never treated as an independent nation) nor in its approach (A pache policy in M exico was designed and implemented mainly by local and state authorities), its object was clearly, al beit indirectly, international: the consolidation of Mexican control over its border with the United States. 


\section{INTRODUCCIÓN}

Uno de los problemas políticos permanentesa los queseha enfrentado México es el desu relación con los pueblosindígenas. En el siglo XIX el problema era tanto más grave cuanto queestaba en juego la definición misma de la nación mexicana. Esta cuestión se complicaba en el caso de las tribus nómadas del septentrión, con las que la relación fue constantemente conflictiva y, a diferencia del centro del país, apenas estaba institucionalizada.

El propósito de este trabajo es analizar, desde la perspectiva de la política exterior, las relaciones de México con los apaches, una deestas tribus nómadas. La tesis aquí manejada es que la política de México hacia los apaches no fueinternacional ni en su objeto (los apaches nunca fueron tratados como una nación independiente) ni en sus instrumentos (la política apache era diseñada y ejecutada principalmente por autoridades locales y estatales). No obstante, esta política sí tenía un objetivo claro - aunque indirecto- de política exterior: consolidar el control mexicano sobresu frontera con los Estados Unidos.

La exposición está dividida en cinco partes. La primera analiza el origen del conflicto. Las siguientes tres tratan las partes dela hipótesis: el estatus de los apaches frente a los mexicanos, el conflicto entre los gobiernos estatales y federal sobre la dirección de la guerra apache y la relación de la política apache con la política exterior. Al final se presentan las conclusiones y los problemas de esta hipótesis.

Esta investigación es un estudio de la política exterior mexicana, sus objetivos y sus problemas en el siglo XIX. Por ello, estetrabajo no es una historia dela guerra apacheen sí misma, y no intenta presentar sino, muy someramente, la perspectiva apache.

\section{LOSORÍGENESDEL CONFLICTO}

Los conquistadores españoles llegaron a la Nueva Vizcaya (losactuales estados de Chihuahua, Coahuila, Durango y Nuevo México) en la segunda mitad del siglo XVI. Sin embargo, la colonización y explotación española dela zona comenzó hasta el siglo XVII, la cual fue, además, siempre precaria debido a las distancias enormes, a la fragilidad delas 
instituciones sociales y políticas recién creadas y a lo reducido de la población (Saravia, 1978 v.1:293-307).

En esta época, el septentrión estaba habitado por diversas tribus nómadas y seminómadas, hostiles a la presencia colonial, como los tepehuanes, los conchos y los tobosos. Los españoles entraron rápidamenteen conflicto con ellasy, tras una revuelta generalizada en 1680, las exterminaron. ${ }^{1}$ La paz resultante y el descubrimiento subsiguientedeminas deplatahicieron del siglo XVIII un siglo próspero para los novohispanos en esta región.

Sin embargo, la expansión estadounidense hacia el oeste -que comenzó a fines del siglo XVIII-, empujó alas tribus nómadas locales al oeste y al sur. Así, los apaches invadieron la Nueva Vizcaya, aprovechando el vacío que provocó el exterminio de los pueblos anteriores (A boites, 1992:29-62). ${ }^{2}$

La convivencia pacífica entre los apaches recién llegados y los españoles ya establecidos resultó imposible. Además de que éstos percibieron a los apaches como invasores, la filosofía política de la dominación española en América insistía en considerar a los indígenas como salvajes, y daba derecho a los conquistadores de someterlos, incluso por la fuerza.(Zavala, 1977:40ss.). El estado de rebeldía en el quese mantuvieron los apaches reforzó estas consideraciones.

Los apaches vivían en tribus nómadas y dependían de la caza y la recolección, que seconvirtieron rápidamente en el robo de cosechas y ganados novohispanos. La habilidad guerrera delos apaches, así como su movilidad, les permitía enfrentarse con ventaja a los rancheros agricultores y ganaderos de la Nueva España, quienes eran mucho más lentos y desorganizados (Griffen, 1988:127ss.).

${ }^{1}$ Para una historia detallada de las rebeliones indígenas de la Nueva Vizcaya en el siglo XVII, véase Saravia, 1978, v.2: primera parte.

${ }^{2}$ Para una historia dela expansión estadounidensehacia el oeste, véase JoséFuentes Mares, Génesis del expansionismo norteamericano, (1984). 
Por otra parte, los apaches estaban divididos en tribus distintas y, en ocasiones, rivales unas de otras. Operaban en territorios muy extensos, pero que seempal maban unos con otros (Villa, 1993), lo que dificultaba a las poblaciones establecidas tratar con ellas, pues los acuerdos alcanzados con una tribu no eran reconocidos por las otrasy, además, eran siempretemporales.

Para defenderse, los novohispanos organizaron un sistema de presidios fronterizos, desde los cuales soldados-agricultores debían impedir las incursiones apaches. También hubo varios intentos - muy costosos- de pacificación. El más importante de ellos tuvo lugar en 1790, impul sado por el virrey Gálvez y su comandantegeneral, cuando un número importante deapaches seasentó al rededor delos presi dios, a cambio de subsidios en especie y privilegios comerciales con las poblaciones cercanas.

La intención del gobierno virreinal no era sólo defender a la Nueva Vizcaya de los estragos de la guerra con los apaches, sino, quizá más importante, consolidar el dominio español sobre el territorio del septentrión, que se encontraba ya amenazado entonces por las ambiciones delas otras potencias europeasy, sobretodo, del expansionismo estadounidense (A boites, 1995:35-58).

El sistema de presidios funcionó eficazmente - al menos por un tiempo-y permitió el desarrol lo de rutas comerciales delargo al cance. La más importante de ellas era la ruta de Santa Fe, que comunicaba a Chihuahua y al resto de la Nueva España con la capital de Nuevo México y, desde allí, con San Luis Missouri y los Estados Unidos. En estecomercio participaban tanto novohispanos como estadounidenses y apaches, eintercambiaban todo tipo de bienes, legal es unos, robados y de contrabando los más (Griffen, 1988:135ss.).

\section{EL ESTATUSDE LOSAPACHES}

El México independienteheredó dela col onia un sistema contradictorio derelaciones con los pueblos indígenas en su seno. La tradición delas leyes de Indias separaba las repúblicas de indios de las de españoles, lo que en el septentrión se expresó en una división tajante entre indígenas sedentarios y nómadas, y en el intento deasentar a éstos en 
presidios o en misiones jesuíticas o franciscanas, alejados de las poblaciones españolas (A boites, 1995:75-89). ${ }^{3}$

Sin embargo, la Constitución de Cádiz de 1812 declaró la igualdad de los habitantes delos reinos españoles y abol ió dichas repúblicas. Estas disposicionesfueron confirmadas por un decreto del imperio mexicano de 1822 y por las constituciones federales de 1824 y 1857 . A lo largo del siglo XIX se tomaron muchas otras disposiciones y medidas para tratar de establecer firmemente esta igualdad. Jurídicamente, los indígenas "formaban partedel organismo nacional" y no existían como entidad separada, aunque, en la práctica, la separación persistía (Lira, 1996:103-118).

La situación delos apaches era aún más confusa, dado su estado de rebel día. A pesar de que en 1834 el gobernador deChihuahua, Joséj. Calvo, sustentó la tesis constitucional deque "[el apache] es hijo dela gran familia mejicanay disfruta delos mismos derechos al suelo donde nació que nosotros como hijos de los conquistadores" (Orozco, 1992:245), y ofreció garantías y seguridades jurídicas a quienes se pacificaran, la opinión generalizada era que se trataba de bárbaros y salvajes, extraños a toda civilización (Orozco, 1992:203-257).

Esta última interpretación se impuso. Un dictamen de 1852 de la Cámara de Diputados federal negaba explícitamente a las tribus nómadas el carácter jurídico de nación; el lo sedebía a que dichas tribus no formaban partedeningún Estado reconocido, por lo tanto, tampoco eran parte dela nación mexicana. ${ }^{4}$ La misma percepción se impuso en los tratados de Guadalupe Hidalgo y de la M esilla, firmados con los

\footnotetext{
${ }^{3}$ Para ver la diferencia en las relaciones entre los indígenas sedentarios (como los yaquis) y los nómadas (los apaches), consúltese Mario Cuevas A rámburu (comp.), Sonora. Textos de su historia, (1989).

4 Dictamen basado en una interpretación de la constitución estadounidense, considerada como fuente dela mexicana. Véaseel "Dictamen presentado a la cámara de Diputados por una comisión de su seno sobre la coalición de los estados de Chihuahua, Coahuila, Durango, Nuevo-León, San Luis, Tamaulipas y Zacatecas, para hacer la guerra a los bárbaros" (Orozco, 1992:279-292).
} 
Estados Unidos en 1848 y 1853, respectivamente, y en los demás documentos oficiales dela época relacionados con el problema. ${ }^{5}$

La aceptación del carácter bárbaro delos apaches autorizó el uso de todos los métodos de guerra, incluidos los prohibidos por el jus belli. ${ }^{6}$ Con esta basese recurrió a la traición, la violación de la inmunidad de losemisariosy la ruptura delas treguas. El objetivo dela guerra apache, sobre todo en la segunda mitad del siglo XIX, fue el exterminio (González, 1995 v.4:24ss.).

\section{ESTADO (MEXICANO) VS. ESTADO (FEDERADO)}

Los estados de la federación - no el Estado mexicano- dirigieron el conflicto con los apaches. A pesar de que el gobierno federal buscó intervenir en varias ocasiones, la falta de capacidad política y de recursos económicos lo obligaron a ceder antelas autoridades estatales (sobre todo en Coahuila, Chihuahua y Sonora).

Traslaguerra deindependencia, quedesquiciólaeconomía nacional al desarticular el comercio, provocar la destrucción y abandono de minas y la desintegración general del país (Zoraida, 1981:768-784), los recursos para la guerra apache dismi nuyeron drásticamente. En estas circunstancias, el viejo sistema novohispano de presidios y establecimientos de paz se derrumbó en 1831.

Los gobiernos locales debieron enfrentar solos la renovación de la amenaza apache, pues sus Ilamados de auxilio al gobierno federal no

\footnotetext{
${ }^{5}$ Véanse, por ejemplo, los tratados de paz, amistad y límites de 1848y de límites de 1853, y el reporte que una comisión pesquisadora entregó al presidente Sebastián Lerdo deTejada en 1872, en Eduardo Enríquez Terrazas y Martha Rodríguez García (comps.), Coahuila. Textos de su historia (1989:143-187).

${ }^{6}$ El jus belli o derecho deguerra es un cuerpo de derecho internacional quecomenzó a compilarse en la segunda mitad del siglo XIX, en las conferencias de La Hayay de Ginebra. Sin embargo, sus provisiones principales, como las de no causar sufrimientos innecesarios, respetar la vida delos prisioneros, respetar a la población no combatiente, etcétera, eran normas de derecho consuetudinario desde muchos siglos atrás (Sorensen, 1973:733-772).
} 
fueron atendidos (sea porque éste los consideró exagerados o porque tenía problemas propios másurgentes). Losgobiernosestatales-tomando atribuciones propias del Estado nacional - declararon formal mente la guerra a los apaches, tomaron las medidas necesarias para enfrentarlos (formación de milicias civiles, entrenamiento de fuerzas regulares, contribuciones especiales) y las financiaron con recursos propios. La estrechez financiera, sin embargo, impidió que estos esfuerzos fueran efectivos (Orozco, 1992:13-31). ${ }^{7}$

También fueron los gobiernos estatales los que negociaron directamente con los apaches y los que firmaron los acuerdos que se alcanzaron. En algunos casos, fueron los pueblos mismos los que, desesperados, pactaron con los apaches. Las distancias con la capital del país e, incluso, del estado, dificultaban cualquier intento de normalización o fiscalización. Casi ninguno de estos acuerdos fue duradero y muchos ni siquiera se pusieron en práctica. ${ }^{8}$

Uno delos pocos acuerdos permanentes fueel quealcanzó el estado de Coahuila con los kikapúes y seminoles poco después del fin dela guerra con los Estados Unidos en 1848, por el que estas dos tribus recibieron tierrasy al gunos derechos en eseestado a cambio deluchar contra las demás tribus nómadas. Sin embargo, incluso este acuerdo se cumplió de manera irregular y dio lugar a muchas críticas y quejas sobre la poca confiabilidad de los apaches (Villarello, 1989:137-143).

En Sonora, el gobierno del estado debió descuidar su atención del problema apache, concentrado en el nortemás despoblado, y enfrentar a los yaquis, un pueblo asentado en las márgenes del río Yaqui y que gozabadeun alto grado decontrol sobresu territorio, fértil y promisorio.

\footnotetext{
${ }^{7}$ Véase como ejemplo deestetipo demovilización el Decreto núm. 122 del gobierno del estado de Sonora del 7 de febrero de 1850.

${ }^{8}$ Estos acuerdos proveían a la sedentarización de los apaches, les permitían una forma limitada de comercio y les obligaba a colaborar en la lucha contra las tribus rebeldes; también incluían provisiones para el intercambio de prisioneros. Los mexicanos sometían estos tratados a la aprobación de los gobiernos estatales y federales y de las autoridades militares, a quienes los apaches deberían supuestamentever, en lo sucesivo, como supremas (W. Griffen, 1988:191ss.).
} 
Los yaquis se sublevaron varias veces entre 1825y 1833y absorbieron la atención política y militar del gobierno de Sonora. Los primeros decretos sonorenses relativos al problema apache aparecieron en la década de 1850, y siguieron la pauta marcada por Chihuahua y Coahuila.(Hu-deH art, 1989:207-213).

La guerra contra los apaches en el siglo XIX, a diferencia de lo que había sido en el siglo XVIII, fue más social que militar, por la desorganización y la pobreza de los esfuerzos gubernamentales. Cada población, ranchería o hacienda quedó prácticamentea merced desus propios recursos. En consecuencia, los apaches pudieron imponer a los mexicanos un sistema de treguas parciales, debido a la vulnerabilidad desus poblaciones y a su dependencia del comercio, tanto con otros mexicanos y estadounidenses, como con los mismos apaches (Griffen, 1988:1-18).

Este sistema era inestabley, además, insatisfactorio desdeel punto devista mexicano del conflicto, pues ponía en duda la solidaridad y la lealtad nacionales. Las autoridades federales comprendieron, poco a poco, los riesgos delasituación, pero fueron incapaces dehacerlefrente, por la magnitud y variedad delos otros problemas quelas agobiaban. ${ }^{9}$

El intento de remediar la dispersión de esfuerzos mediante una coal ición de estados fronterizos fracasó por la oposición del Congreso dela Unión. A pesar de quela Cámara de Diputados había aprobado dicha coalición en abril de 1852, el senado la rechazó en diciembre, arguyendo el peligro para la integridad territorial del país. El senado se negó, además, a aprobar fondos nuevos y exigió que el gobierno federal dirigiera la guerra apache. Por otra parte, la lentitud con que fue tratado el problema demuestra lo secundario que esta cuestión resultaba para las autoridades federales. ${ }^{10}$

\footnotetext{
${ }^{9}$ Véase, por ejemplo, la carta del secretario de Guerra y Marina al de Relaciones Exteriores de marzo de 1848, citada por I. Villarello Vélez, (1989).

10 "Dictamen de la segunda comisión de guerra a la cámara de senadores sobre el acuerdo relativo a la coalición de estados fronterizos para la defensa contra los bárbaros", en: Orozco (1992:293-300).
} 
Por otra parte, a todo lo largo del siglo XIX, los gobiernos estatales y federal se vieron envueltos en una disputa sobre el destino de los escasos recursos federal es para la guerra apache. El gobierno federal defendía la creación de colonias militares y penales, controladas directamente por él, mientras que los gobiernos estatales preferían recibir estos recursos en efectivo, para disponer de ellos según su criterio. Dada las dificultades materiales para organizar y sostener las colonias, la opción estatal -de organizar milicias y preparar a los pobladores para que hicieran frentepor cuenta propia a la amenazaseimpuso (González, Cosío y Monroy, 1955:176-190).

Aún después delaintervención francesa (1862-1867), cuando el Estado mexicano empezó a consolidarse demanera definitiva, la dirección dela guerra apache siguió en manos estatales. Así, las recompensas por cabelleras y cautivos apaches (un expediente diseñado para garantizar la participación privada en la guerra) se pagaban con recursos estatales —o federales, controlados por los estados (Calderón, 1955:321,359).

La disputa entreestados y federación llegó hasta el final dela guerra apache. En consecuencia, el ejército federal senegó a participar, a pesar deestar en posición dehacerlo, en la batal la de Tres Castillos, en 1882, cuando las fuerzas públicas del estado de Chihuahua derrotaron a la última banda apache (A boites, 1992:113ss).

\section{POLITICA APACHEY POLÍTICA EXTERIOR}

Ya desdeel periodo colonial las autoridades novohispanas advertían que la integridad del territorio dependía de la ocupación efectiva del septentrión mexicano y que ésta, a su vez, dependía dela pacificación o el exterminio delosapaches. El proyecto ilustrado definales del siglo XVIII buscaba hacer permanente la presencia militar, política y económica novohispana en la Nueva Vizcaya, con la instalación de colonias y presidios. Este proyecto fracasó tras la independencia de México por falta derecursos; no obstante, siguió sirviendo demodelo e inspiración para los gobiernos posteriores. De cual quier manera, el desorden quereinaba en el país, en general, y en los estados fronterizos, en particular, dificultó cual quier avancey, en la práctica, complicó aún máslasituación. 
A partir de 1831, la situación se volvió crítica. Los ataques de los apaches perjudicaron gravementelas bases del sistema económico del septentrión - sobre todo la ganadería- e indujeron el abandono de poblaciones importantes, sobre todo en Sonora y Chihuahua. Las comunicaciones se volvieron inseguras e incluso la minería, otrora pujante, se desquició (A boites, 1995:35-38).

La guerra con los Estados Unidos (1846-1848) y la pérdida de los territorios más septentrionales demostraron que los temores al expansionismo estaban plenamente fundados y que la solución del problema era urgente. El poblamiento de los territorios fronterizos seguía siendo indispensable (Aboites, 1995).

La compra dela M esilla en 1853 y las pretensiones estadounidenses deperseguir "en cal iente" (hot pursuit) a los apaches, violando incluso territorio mexicano, demostraron la persistencia de la amenaza. Este problema no seresolvió formal mentesino en 1882, cuando un acuerdo autorizó a ambos países - y no sólo a los Estados Unidos- a cruzar la frontera en persecución de los apaches y el gobierno estadounidense abandonó oficial mentetoda pretensión sobre territorio mexicano. Sin embargo, y a pesar deestas fricciones, existían acuerdos informales de cooperación y trabajo conjunto entrelas autoridades locales deambos lados de la frontera (Lajous, 1990:47ss).

El gobierno federal trató departicipar en la solución del problema. El citado dictamen de la Cámara de Diputados de 1852 decía: "si importa convertir los desiertos en poblaciones, más imperiosa debeser la obligación deimpedir quelas poblaciones se conviertan en desiertos" (Orozco, 1992:285). Por ello la cámara se negó a eliminar los recursos destinados a la col onización mil itar y civil, ni siquiera para transferirlos a otras formas delucha contra los apaches. El senado también sostuvo esta posición (Orozco, 1992:279-303).

Por otra parte, en lasnegociaciones del Tratado deGuadalupeH idal go -que terminó la guerra con los Estados Unidos-, México logró la inclusión de una cláusula que responsabilizaba a los estadounidenses de las incursiones apaches en México provenientes de su territorio, a las que debía reprimir o reparar. ${ }^{11}$ La anulación deesta cláusula -que fue total mente inoperante- por el Tratado de la M esilla fue objeto deuna reparación pecuniaria (Zoraida, 1990:142, 154, 168). ${ }^{12}$ 
Las secretarías federales de Guerra y Marina y de Relaciones Exteriores, buscaron coordinarseentresí y con la Legación de México en los Estados Unidos para comprar armas y exigir extradiciones y liberación de prisioneros a las autoridades estadounidenses. Estas actividades consumieron buena parte del tiempo de los ministros mexicanos en Washington, casi hasta el final del siglo XIX.13

En el plano estatal, al gunas de las disposiciones más importantes estuvieron destinadas a socorrer a las poblaciones más expuestas a las incursiones apaches. Las medidas comprendían desdela exención de diezmos e impuestos, hasta la instalación de cuarteles y presidios en dichos poblados (Enriquez y Rodríguez, 1989:203-257).

Todo el sistema de distribución y tenencia de la tierra en el norte del país giró al rededor de la necesidad de poblar el territorio. Los estados y la federación también se disputaron el control del proceso (que, en estecaso, ganó el gobierno federal ), pero no pusieron en duda la premisa básica del poblamiento (A boites, 1992:95-119). Por otra parte, varios proyectos de promoción a la inmigración incluían, entre las obligaciones inmediatas de los migrantes - generalmente exentos de toda imposición durante cinco años- el al istarse para combatir a los nómadas (Calderón, 1955:74).

La relación entre la colonización del septentrión y la lucha contra las tribus nómadas quedó demostrada con la introducción del ferrocarril. Éste propició, al mismo tiempo, la represión definitiva de losapachesy el movimiento depoblación hacia el norte(principalmente Chihuahua, Nuevo León y Sonora) (González N avarro, 1995:25ss).

\footnotetext{
${ }^{11}$ Esta cláusula fuesugerida por la legislatura deChihuahua, quebuscaba garantizar la seguridad de su población. Véase “Demanda de la diputación permanente del Congreso en relación al tratado de paz con Estados Unidosy los indios 'bárbaros' “, en Orozco, (1992:256). y el art. XI del Tratado de paz, amistad y límites del 2 de febrero de 1848.

${ }^{12}$ Véansetambién los arts. II y III del Tratado delímites del 30 de diciembre de 1853. ${ }_{13}^{13}$ Por ejemplo, el reportecitado dela comisión pesquisadora al presidenteLerdo de Tejada en 1872, en E. Enríquez Terrazas y M. Rodríguez García (comps.), Coahuila, Textos de su historia (1989:143-187).
} 
El ferrocarril y los demás sistemas de comunicaciones y transportes introducidos a partir de 1867, integraron el septentrión, política y económicamente, al resto del país de manera permanente. México se consolidó como Estado nacional en esteperiodo y disminuyó, al mismo tiempo, la amenaza deseparación territorial delos estados fronterizos.

\section{OTROS ELEM ENTOS DEL PROBLEM A}

Queda claro quela consolidación del control mexicano sobresu frontera norte estuvo directamente relacionada con la solución del problema apache, y queesta solución estuvo básicamenteen manos delos gobiernos estatales, a pesar de su importancia política para la federación. También es evidente que la nación apache nunca fue tratada como tal por las autoridades mexicanas, quienes finalmente la exterminaron.

Sin embargo, este análisis no es perfecto. Por un lado, el interés estatal por terminar con la amenaza apache no estuvo completamentedeterminado por el deseo deconsol idar lafrontera, sino también -sobretodo a partir de1850 - por el interés delas oligarquías locales, terratenientes y ganaderas, en acabar con la amenaza más importante alafuentedesu riqueza (el caso más notableesel delafamilia Terrazas en Chihuahua) (Orozco, 1992; A boites, 1992).

Por el otro, el Estado mexicano sí se ocupó del problema apachey del problema general dela frontera- por las vías tradicionales dela política exterior. Ello quedó demostrado en quela mayor parte delas fricciones diplomáticas entreMéxico y los Estados Unidos en la segunda mitad del siglo XIX, tuvieron como trasfondo el problema de las incursiones nómadas.

Otro factor que debe tomarse en cuenta es la participación estadounidenseen la solución del problema apachemexicano. A unque al principio hubo una especie de alianza entre apaches y estadounidenses contra los mexicanos (que eran el enemigo común), poco a poco las autoridades estatales y federal de los Estados Unidos intervinieron en el conflicto y declararon la guerra a los apaches por su cuenta (Nuevo México, por ejemplo, lo hizo en 1854) (Antochiw, 1989:405ss). 
Después del término de la guerra de secesión (1865), los Estados Unidos pudieron enviar un número importantedetropas a la frontera con M éxico, con el objeto de acabar con los apaches y las otras tribus nómadas insumisas, creándoles un segundo frente. El avance tecnológico y estratégico al que no tuvieron acceso los apaches, por razones tanto políticas como culturales, fueotro elemento en su derrota (González y González, 1955:185ss).

También conviene reconsiderar el grado de control que sealcanzó sobre la frontera. México nunca pudo impedir las expediciones punitivas estadounidenses en contra delos apaches o deotras personas. Formalizando una situación de facto, México y los Estados Unidos firmaron un acuerdo en julio de 1882, que permitía a las fuerzas federales de cada país el paso recíproco de la frontera en persecución de las tribus nómadas (u otros delincuentes) (González Navarro, 1995:244).

A pesar de estos problemas, la política exterior no tradicional, conducida por entidades subestatales (estados federados) frentea un enemigo extracivilizacional, "bárbaro", esfundamental en la explicación delafrontera septentrional mexicana como la conocemos hoy. Así, una perspectiva heterod oxa de los problemas de México en sus relaciones con las naciones indígenas puede servir para analizar exitosamentela política exterior mexicana.

\section{BIBLIOGRAFÍA}

A boites A guilar, Luis (1992). Breve historia deChihuahua, El Colegio de México-Fondo deCultura Económica, México.

- (1995) N orte precario. P oblamiento y colonización en M éxico (17601940), México, El Colegio deM éxico-CIESAS.

Antochiw, Michel (1989). "La guerra apache", en Mario Cuevas Arámburu (comp.), Sonora. Textos desu historia, México, Gobierno del estado de Sonora-Instituto M ora, vol. 2, pp. 405-407.

Calderón, Francisco (1955) “La república restaurada. La vida económica", en Daniel Cosío Villegas (dir.), H istoria moderna de M éxico, México, Hermes, vol. 2. 
Cuevas A rámburu, Mario (comp.) (1989). Sonora. Textos de su historia, en tres volúmenes, México, Gobierno del estado de SonoraInstituto Mora.

Decreto núm. 122 del gobierno del estado de Sonora, 7 de febrero de 1850. Enríquez Terrazas, Eduardo y Martha Rodríguez García (comps.) (1989). Coahuila. Textos desu historia, México, Gobierno del estado deCoahuila-Instituto Mora.

Fuentes Mares, José (1984). Génesis del expansionismo norteamericano, México, El Colegio deMéxico.

González Navarro, Moisés (1955). "El porfiriato. La vida social”, en

Daniel Cosío Villegas (dir.), H istoria moderna de M éxico, México, Hermes, vol. 4.

González y González, Luis, Emma Cosío Villegasy Guadal upeM onroy (1955). "La república restaurada. La vida social", en Daniel Cosío Villegas (dir.), H istoria moderna deM éxico, México, Hermes, vol. 3. Griffen, William (1988). U tmost Good Faith. Patterns of A pache-M exican $\mathrm{H}$ ostilities in N orthern Chihuahua, 1821-1848, Albuquerque, University of New Mexico Press.

Hu-deHart, Evelyn. (1989). “La relación entrela fuerza y la política en la revolución de los yaquis. La época de Banderas, 1825-1833", en M. Cuevas A rámburo (comp.). Sonora. Textos de su historia, vol. 1, M éxico, Gobierno del estado de Sonora-Instituto M ora.

Lajous, Roberta (1990). M éxico y el mundo. Historia de sus relaciones exteriores, México, Senado dela República, vol. 4.

Lira González, Andrés (1996). “La extraña anomalía. Realidades indígenas en el México del siglo XIX", en Fernando Benítez, Carmen Cordero, et al., Cultura y derechos de los pueblos indígenas en M éxico, México, Archivo General de la Nación-Fondo de CulturaEconómica, pp.103-118.

Orozco, Víctor (1992). Las guerras indias en la historia de Chihuahua. A ntología, Ciudad Juárez, UACJ-Instituto Chihuahuense de la Cultura.

Saravia, A tanasio (1978). A puntes para la historia de la Nueva Vizcaya, México, UNAM, vols. 1 y 2.

Sorensen, Max (1973). M anual de derecho internacional público, trad. Dotación Carneige para la Paz, México, FCE. 
Tratado de límites, 30 de diciembre de 1853.

Tratado de paz, amistad y límites, 2 de febrero de 1848.

Vásquez, JosefinaZoraida (1981). “Los primeros tropiezos”, en Daniel Cosío Villegas (ed.), H istoria general deM éxico, México, El Colegio deMéxico, pp. 768-784.

_ (1990). M éxico y el mundo. Historia de sus relaciones exteriores, México, Senado dela República, vol. 1.

Villa, Guadalupe (1993). ¡A paches! , M éxico, Instituto M ora.

Villarello Vélez, Ildefonso (1989). "De los presidios a las colonias militares", en Eduardo Enríquez Terrazas y Martha Rodríguez García (comps.), Coahuila. Textos desu historia, México, Gobierno del estado de Coahuila-Instituto Mora, pp. 137-143.

Zavala, Silvio (1977). La filosofía política en la conquista deA mérica, México, Fondo de Cultura Económica.

Zoraida Vásquez, Josefina (1981). “Los primeros tropiezos” en Daniel Cosío Villegas (ed.), H istoria general deM éxico, México, El Colegio deMéxico.

(1990). M éxico y el mundo. Historia de sus relaciones exteriores, México, Senado dela República, vol. 1. 